\title{
Congenital Lamellar Icthyosis (Collodion Baby)
}

\section{Baghel $\mathrm{B}^{1}$}

${ }^{1}$ Dr. Bhagat Baghel, MBBS. MD, Assistant Professor, Department of Paediatrics, Government Medical College Jagdalpur (Bastar), Chattisgarh-494001, India

Address for correspondence: Dr. Bhagat Baghel, E-mail: bhagatbaghel75@yahoo.com

\begin{abstract}
Lamellar ichthyosis, also known as ichthyosis lammellaris and nonbullous congenital ichthyosis, is a rare inherited skin disorder, affecting around 1 in 600,000 people the images of this rare variety of skin disorder has been presented here.
\end{abstract}

\section{The Case}

A female baby was delivered per vaginaly and baby had cried immediately after birth. The weight was $2.50 \mathrm{~kg}$, length $46 \mathrm{~cm}$ and head circumference was 32 $\mathrm{cm}$. Affected baby was born in a membrane with a shiny waxy outer layer to the skin (Fig 1). It sheded in 5-8 days, revealing extensive scaling of the skin. With increasing age, the scaling was concentrated around joints in areas such as the groin, the armpits, the inside of the elbow and the neck. The scales tiled the skin and resembled fish scales. This was the 3 rd sibling, first two still alive and healthy; there was no history of skin disorders in any family member. The eyelids and mouth had the appearance of being forced open due to the tightness of the skin. This was also associated with eversion of the eyelids (ectropion) Fig 2. In the initial few days the baby was not able to feed breast milk and which was because of excessive tightness of skin around oral cavity, later on the baby was discharged from NICU as the baby was able to feed breast milk.

The term collodion baby applies to newborns that appear to have an extra layer of skin (known as a collodion membrane) that has a collodion like quality. The appearance is often described as a shiny film looking like a layer of vaseline and extensive scaling of the skin caused by hyperkeratosis.

Collodion babies can have severe medical consequences, mainly because the baby loosing heat and fluid through the abnormal skin. This can lead to hypothermia and dehydration. Strategies to prevent these problems are; use of emollients or nursing the baby in a humidified incubator. There is also an increased risk of skin infection and mechanical compression leading to problems like limb ischemia. The condition is not thought to be painful or in itself distressing to the child. Nursing usually takes place in a neonatal intensive care unit, and good intensive care seems to improve the prognosis markedly.

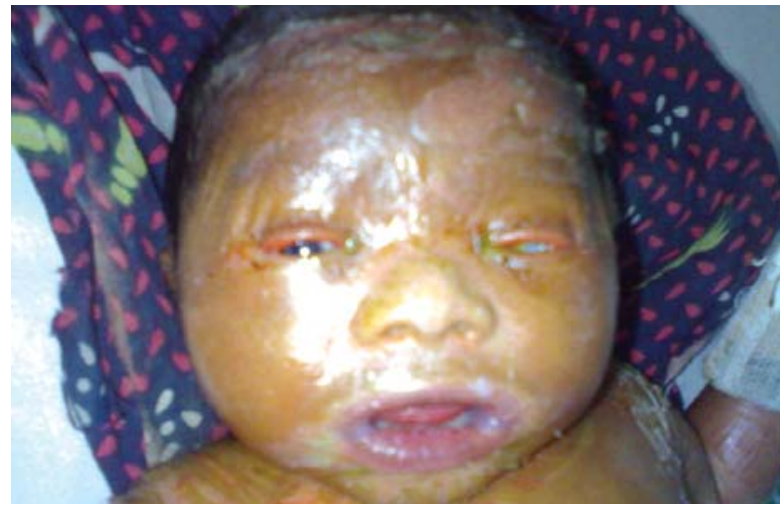

Fig 1: Photograph of the face of the baby with eversion of the eyelids (ectropion)

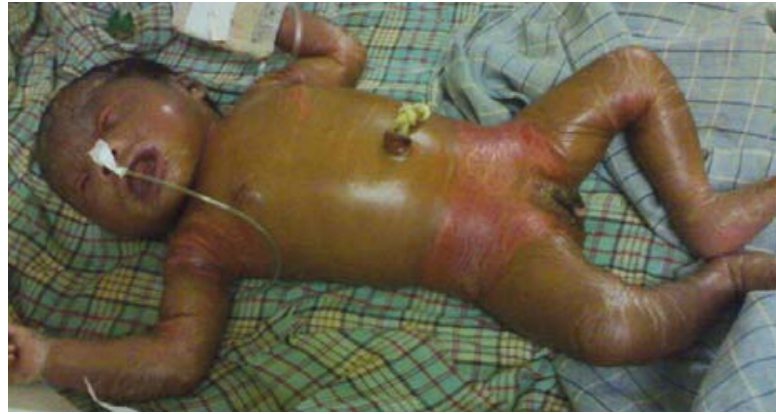

Fig 2: Showing the baby with shiny waxy outer layer to the skin

How to cite this article?

Baghel B. Congenital Lamellar Icthyosis (Collodion baby). J Nepal Paediatr Soc 2011;31(3):257 